\title{
Full-Term Abdominal Pregnancy with Dead Fetus: A Case Report
}

Harshida Chaudhari ${ }^{1 *}$ and Nilam Prajapati ${ }^{2}$

${ }^{1}$ Montana State University, Bozeman, Montana, USA

${ }^{2} \mathrm{New}$ civil Hospital and Government Medical College, Surat, India

\begin{abstract}
Introduction: Abdominal pregnancy is a rare form of ectopic pregnancy and is frequently misdiagnosed. Here, we report a full-term abdominal pregnancy with dead fetus in a case of second gravida. The diagnosis was missed despite regular antenatal care and was diagnosed intraoperatively.

Case presentation: A 25 year old second gravida with a previous caesarean section was referred to us from a private hospital as a case of 38-week pregnancy with ruptured uterus. She had no other positive history except for an abdominal pain for 12 hours and loss of fetal movements for 6 hours. On examination, the abdomen was distended and tender, the uterus was well contracted and was palpable at the level of umbilicus and superficial fetal parts were felt. There were no fetal heart sounds and fetal movements were absent. On pelvic examination, the cervix was posteriorly placed with uneffaced and closed cervical os. Our patient underwent emergency exploratory laparotomy under spinal anesthesia and was found to have an intact, well-contracted uterus with the dead fetus lying in the peritoneal cavity surrounded by an intact amniotic sac. The placenta was attached to the greater omentum. The baby was extracted uneventfully. Mother had profuse blood loss during the procedure due to separation of abnormally implanted placenta. She was resuscitated with intravenous fluid and blood products. The postoperative period was uneventful and she recovered completely.
\end{abstract}

Conclusion: Abdominal pregnancy poses special challenges to obstetricians working in remote areas with limited resources for diagnosis. A high index of suspicion is required in recognizing this condition in order to prevent adverse maternal and fetal outcomes.

Keywords: Ectopic pregnancy; Abdominal pregnancy; Extra-uterine term pregnancy

\section{Introduction}

Abdominal pregnancy is an extremely rare form of extra-uterine pregnancies. It constitutes about $1 \%$ of ectopic pregnancies [1]. Abdominal pregnancy can be primary where the fertilized ovum implants itself on an abdominal organ. Most of the abdominal pregnancies are secondary where fertilized ovum first implants in the uterus, ovary or fallopian tube. It subsequently implants on a peritoneal surface following tubal abortion or uterine scar rupture [2]. The maternal mortality rate for women with an abdominal pregnancy is as high as $18-20 \%$ owing to the risk of catastrophic hemorrhaging from placental separation $[1,2]$. The chances of survival of a newborn are very low with the perinatal mortality rate being $40-95 \%$ [3]. Abdominal ectopic pregnancy is frequently missed prior to an operative intervention leading to dramatic complications for both mother and fetus [4]. Most cases of advanced ectopic pregnancy are associated with a dead fetus, and the ones who survive face numerous challenges due to congenital malformations [5]. Here we present a case of full-term abdominal ectopic pregnancy with dead fetus and its management.

\section{Case Report}

A 25 year old second gravida with a previous caesarean section was referred to us from a private hospital as a case of 38-week pregnancy with ruptured uterus. She had a history of abdominal pain for 12 hours and loss of fetal movements for 6 hours. There was no history of bleeding or leaking per vagina. She had had regular antenatal care from a primary health care center in a remote area. She was on regular multivitamin and iron tablets throughout her pregnancy and was given two doses of tetanus toxoid. She had not had an ultrasound performed during this pregnancy as the ultrasound facility was not available to the local clinic where she went for her antenatal care. Her antenatal period was uneventful until the $38^{\text {th }}$ week when she started having abdominal pain. She had no significant past history except for one caesarean section during her first pregnancy that was performed for a fetal distress in the first stage of labor. She had no history of any other abdominal surgeries, contraceptive use, primary or secondary infertility, sexually transmitted diseases or pelvic inflammatory diseases.

On examination, she looked anxious. She had a pulse rate of $104 / \mathrm{min}$, blood pressure of $120 / 80 \mathrm{~mm} \mathrm{Hg}$ and mild pallor. Her cardiovascular and respiratory system did not reveal any abnormalities. The abdomen was distended and tender. The uterus was well contracted and was palpable at the level of umbilicus. The superficial fetal parts were felt. There were no fetal heart sounds and fetal movements were absent. On pelvic examination, the cervix was posteriorly placed with unaffected and closed cervical. There was no blood or fluid leaking from the vagina. The uterus felt bulky and appeared separate from the fetus. Provisional diagnosis of ruptured uterus due to scar dehiscence was made.

Her laboratory results showed a normal complete blood count except hemoglobin of $9.8 \mathrm{gm} / \mathrm{dl}$. Her liver function tests, renal function tests, serum electrolytes and coagulation profile were within normal limits. Patient was shifted to an operating room for emergency exploratory laparotomy. Also, her blood sample was sent for blood group and cross match and a blood bank was informed to keep four units of blood ready. Her abdomen was opened with a midline vertical

*Corresponding author: Harshida Chaudhari, 103 Paisley Court, Apt. E, Bozeman MT 59715, USA, Tel: +1- 406-551-4959; E-mail: harshidajaydeep@gmail.com

Received September 14, 2012; Published October 18, 2012

Citation: Chaudhari H, Prajapati N (2012) Full-Term Abdominal Pregnancy with Dead Fetus: A Case Report. 1:434. doi:10.4172/scientificreports.434

Copyright: (c) 2012 Chaudhari $\mathrm{H}$, et al. This is an open-access article distributed under the terms of the Creative Commons Attribution License, which permits unrestricted use, distribution, and reproduction in any medium, provided the original author and source are credited. 
incision under spinal anesthesia. On opening a peritoneal cavity, a dead fetus was found in the peritoneal cavity with an intact amniotic sac. Uterus was 20 weeks in size, firm and well contracted, and there was no scar dehiscence. Both ovaries and fallopian tubes were morphologically normal. Intraoperative diagnosis of abdominal ectopic pregnancy was made and the decision was made to extend the incision above the umbilicus. While extending the incision, injury to placenta led to massive blood loss. Patient went into severe hypotension. Four units of blood were transfused, general anesthesia was given and the patient was intubated. The incision was extended above the umbilicus. The placenta was found to be attached to the greater omentum. A $2.7 \mathrm{~kg}$, full-term dead, male fetus was delivered. On examination, the fetus had congenital talipes equinovarus and polydactyly. Absence of macerative changes was indicative of a recent fetal death. We were not able to assess the internal anomalies as the mother refused to give consent for an autopsy. Liquor was thick; meconium stained. The placenta was separated from the greater omentum and removed in piecemeal. Peritoneal wash was given with saline and a drain was placed in situ. The abdomen was closed in layers. Patient was given broad spectrum intravenous antibiotics for two days followed by oral antibiotics for five days. Postoperative course was uneventful. Breast milk suppression was done with a dopamine agonist (Cabergoline $0.5 \mathrm{mg} 2$ tablets stat). Patient was discharged on the seventh day after suture removal. Contraceptive counseling was done.

\section{Discussion}

Abdominal pregnancy is a rare form of ectopic pregnancy. It is associated with high maternal morbidity and mortality rate. Morbidity is due to bleeding, infection, toxemia, DIC, anemia and pulmonary embolism. The perinatal mortality rate is $40-95 \%$ [2]. Birth defects have been found in about $21 \%$ of babies due to compression of the fetus in the absence of amniotic fluid buffer. Typical deformities include facial and cranial asymmetry, limb defects, joint abnormalities and CNS malformations [3]. Several factors have been identified that increase the risk of ectopic pregnancy such as history of pelvic inflammatory disease, chlamydial or gonococcal cervicitis, infertility, morphological tubal abnormality, previous tubal surgery, recent use of Intrauterine Device (IUD) as contraception and history of previous ectopic pregnancy [6].

Ultrasound examination is a versatile and safe imaging modality to diagnose ectopic pregnancy. However, ultrasound facilities are still inaccessible to many who live in remote areas. The hormonal environment in ectopic pregnancy can produce an intrauterine fluid collection that mimics the gestational sac. Visualization of a gestational $\mathrm{sac}$ on a transvaginal ultrasound cannot confirm intrauterine pregnancy. Identification of an intrauterine gestational sac with yolk sac on a transvaginal ultrasound during early pregnancy rules out ectopic pregnancy in most patients. This finding should be reinforced by threshold $\beta$-hCG level (1000-2000 IU/L with transvaginal approach, $6500 \mathrm{IU} / \mathrm{L}$ with transabdominal approach) at which an intrauterine gestational sac can be reliably seen in normal pregnancy [6].

Advanced abdominal pregnancy can pose diagnostic difficulties due to variable clinical presentation. A high index of suspicion is required in making a prompt diagnosis especially in low resource centers [5]. Several clinical features such as persistent abdominal pain, painful fetal movements, palpation of an abdominal mass separate from the uterus, vaginal bleeding, or unaffected and posteriorly displaced pinpoint cervical os should raise suspicion [7]. However, our patient did not have any symptoms during her antenatal period except for abdominal pain, which started at 38 weeks. Ultrasound can be very helpful in earlier stages of pregnancy but can be disappointing in later stages. Absence of a uterine wall surrounding the fetus, fetal parts very close to the abdominal wall and abnormal lie on ultrasound examination should raise suspicion for abdominal ectopic in advanced pregnancy [3]. High maternal serum Alpha-fetoprotein levels and lack of myometrial response to oxytocin stimulation are other tools to help diagnose abdominal pregnancy [8].

Once diagnosed, the widely accepted treatment is immediate laparotomy due to risk of complications. However, controversy exists regarding the use of a conservative approach if pregnancy has been discovered to be in an advanced stage and the fetus is alive. A conservative approach should only be undertaken if the patient can be monitored closely, preferably in hospital $[3,7]$. Another issue of debate is whether to remove the placenta during laparotomy. Unless the blood supply to the placenta is easily identified and secured, it is preferable to leave it in situ as attempts to remove it can result in catastrophic hemorrhage due to abnormal implantation $[3,4]$. Total regression of placental function is believed to occur within four months if the placenta is not removed during surgery. Patient should be followed up with serial human chorionic gonadotrophin levels during this period $[1,5]$. If a placenta does not resorb spontaneously, there is an increased risk of hemorrhage, sepsis and necrosis and a patient might need to undergo another surgery [4]. In our case, significant bleeding from the detached portion of the placenta prompted its removal to secure hemostasis.

\section{Conclusion}

In the above case abdominal pregnancy was carried up to term without diagnosis in spite of regular antenatal care. An advanced abdominal pregnancy is difficult to diagnose and high index of suspicion is required by the clinician. A clinician should be very vigilant in diagnosing this condition and should ask for help from more experienced clinicians when in doubt.

\section{References}

1. Yildizhan R, Kolusari A, Adali F, Adali E, Kurdoglu M, et al. (2009) Primary abdominal ectopic pregnancy: a case report. Cases Journal 2: 8485.

2. Hamid AN, Begum R, Sultana Z, Akhter N (2010) Advanced Abdomina Pregnancy: A Case Report. Journal of Chittagong Medical College Teachers' Association 21: 74-76.

3. Dahab A, Aburass R, Shawkat W, Babgi R, Essa O, et al. (2011) Full-term extrauterine abdominal pregnancy: a case report. J Med Case Rep 5: 531

4. Nunyalulendho DN, Einterz EM (2008) Advanced abdominal pregnancy: case report and review of 163 cases reported since 1946. Rural and Remote Health 8: 1087.

5. Baffoe P, Fofie C, Gandau BN (2011) Term Abdominal Pregnancy with Healthy Newborn: A Case Report. Ghana Med J 46: 81-83.

6. Murray H, Baakdah H, Bardell T, Tulandi T (2005) Diagnosis and treatment of ectopic pregnanacy. CMAJ 173: 905-912.

7. Isah AY, Ahmed Y, Nwobodo El, Ekele BA (2008) Abdominal Pregnancy with A Full Term Live Fetus: Case Report. Ann Afr Med 7: 198-199.

8. Ludwig M, Kaisi M, Bauer O, Diedrich K (1999) Case Report: The forgotten child-a case of heterotopic, intra-abdominal and intrauterine pregnancy carried to term. Hum Reprod 14: 1372-1374. 